Trinity University

Digital Commons @ Trinity

Chemistry Faculty Research

Chemistry Department

$12-2016$

\title{
Cucurbit[7]uril-Tetramethylrhodamine Conjugate for Direct Sensing and Cellular Imaging
}

Andrew T. Bockus

TrinityUniversity, abockus@trinity.edu

Lauren C. Smith

Trinity University, 1smith16@trinity.edu

Amy G. Grice

TrinityUniversity, agrice@trinity.edu

Omar A. Ali

Trinity University, oali@trinity.edu

Carolyn C. Young

Trinity University, cyoung1@trinity.edu

See next page for additional authors

Follow this and additional works at: https://digitalcommons.trinity.edu/chem_faculty

Part of the Chemistry Commons

\section{Repository Citation}

Bockus, A. T., Smith, L. C., Grice, A. G., Ali, O. A., Young, C. C., Mobley, W., .. . Urbach, A. R. (2016). Cucurbit[7]uriltetramethylrhodamine conjugate for direct sensing and cellular imaging. Journal of the American Chemical Society, 138(50), 16549-16552. doi:10.1021/jacs.6b11140 
Authors

Andrew T. Bockus, Lauren C. Smith, Amy G. Grice, Omar A. Ali, Carolyn C. Young, William Mobley, Ashley Leek, James Lewis Roberts, Brittany Vinciguerra, Lyle D. Isaacs, and Adam R. Urbach 


\section{Cucurbit[7]uril-Tetramethylrhodamine Conjugate for Direct Sensing and Cellular Imaging}

Andrew T. Bockus, ${ }^{\text {a }}$ Lauren C. Smith, ${ }^{\mathrm{a}}$ Amy G. Grice, ${ }^{\mathrm{a}}$ Omar A. Ali, ${ }^{\mathrm{a}}$ Carolyn C. Young, ${ }^{\mathrm{a}}$ William Mobley, ${ }^{a}$ Ashley Leek, ${ }^{b}$ James L. Roberts, ${ }^{b}$ Brittany Vinciguerra, ${ }^{c}$ Lyle Isaacs, ${ }^{c}$ and Adam R. Urbach ${ }^{a *}$

${ }^{a}$ Department of Chemistry, Trinity University, 1 Trinity Place, San Antonio, TX 78212, USA

${ }^{b}$ Department of Biology, Trinity University, 1 Trinity Place, San Antonio, TX 78212, USA

${ }^{c}$ Department of Chemistry and Biochemistry, University of Maryland, College Park, MD, 20742, USA

ABSTRACT: This paper describes the design and synthesis of a conjugate (Q7R) comprising the synthetic host cucurbit[7]uril (Q7) linked to the fluorescent dye tetramethylrhodamine (TMR), and the characterization of its optical and guest-binding properties as well as its cellular uptake. Q7R was synthesized in two steps from monofunctionalized azidobutyl-Q7 and NHS-activated TMR. The fluorescence of Q7R is quenched upon guest binding, and this observable was used to determine equilibrium dissociation constant $\left(\mathrm{K}_{\mathrm{d}}\right)$ values. Unexpectedly, the $\mathrm{K}_{\mathrm{d}}$ values for guests binding to Q7R and to unmodified Q7 were essentially identical. Therefore, Q7R can directly report binding to Q7 without an energetic penalty due to the conjugated fluorophore. This result demonstrates a potentially general strategy for the design of single-component host-indicator conjugates that respond sensitively to analytes without perturbing the binding properties of the host. The unique properties of Q7R enabled measurement of $K_{d}$ values across three orders of magnitude and at concentrations as low as $0.7 \mathrm{nM}$. This result is particularly relevant given the unmatched range of guests and binding affinities demonstrated for Q7. Confocal fluorescence microscopy of live and fixed HT22 neurons revealed the cellular uptake of Q7R and its punctate localization in the cytoplasm. Q7R did not alter cell growth at concentrations up to $2.2 \mu \mathrm{M}$ over four days. These experiments demonstrate the feasibility of Q7R as a direct sensor for guest binding and as a cell-permeable compound for imaging applications. 


\section{INTRODUCTION}

The development of novel fluorescent compounds and fluorescence-based assays continues to drive advancements in many areas of basic and applied science. In particular, the field of supramolecular chemistry has benefited from the practical use of fluorescent dyes to measure the thermodynamics of host-guest binding and the kinetics of enzymatic reactions. ${ }^{1}$ These assays rely on an indicatordisplacement strategy, in which the target analyte competitively displaces a fluorescent guest, or vice versa, and results in a change in fluorescence intensity. ${ }^{2}$ Indicator displacement assays (IDAs) do not require the covalent modification of host or guest, thus preserving their binding properties and obviating the need for chemical synthesis. The noncovalent association of host and indicator, however, necessitates the tuning of their binding affinity and working concentrations in order to ensure competitive binding conditions. Most hosts have a limited range of affinities and thus a limited range of working conditions for competitive binding assays. ${ }^{3}$ Moreover, IDAs have limited utility under continuous flow conditions (e.g., flow sensing or separations) or in biological imaging applications in which the working concentrations are well below the $\mathrm{K}_{\mathrm{d}}$ value of the host・indicator complex. ${ }^{2}$

Covalent conjugation of a host to an indicator removes the dependence of their association on concentration. Careful design of a conjugate can yield single-component, direct sensors capable of detecting guest binding over a wide range of concentrations. ${ }^{4-8}$ Covalent modification, however, is likely to alter the host binding properties. Given the narrow scope of binding affinities available to most host compounds, the advantages of covalent host-indicator conjugates do not typically justify the additional effort required for design, synthesis, characterization, and optimization. One synthetic receptor, cucurbit[7]uril (Q7), may be an exception.

Q7 is a barrel-shaped, water-soluble, organic macrocycle composed of seven glycoluril units linked by pairs of methylene groups. ${ }^{9-13}$ Binding is driven by displacement of frustrated water molecules and electrostatic attraction of cationic groups on the guest to $\mathrm{C}=\mathrm{O}$ dipoles on the host. ${ }^{14} \mathrm{Q} 7$ is unique among synthetic receptors in the breadth of its guests and the corresponding range of $K_{d}$ values, which span the millimolar to attomolar range in aqueous solution. ${ }^{15-18}$ Accordingly, Q7 has found many applications, including sensing, separation, catalysis and drug delivery, to name a few. ${ }^{11-13,17,19-21}$

There are many fluorescent guests for Q7, ${ }^{1}$ and myriad IDA-based studies of Q7 have been reported. Q7 is difficult to modify covalently, ${ }^{22}$ but recent synthetic approaches to monofunctionalization have enabled the synthesis of several discrete conjugates. ${ }^{23-27}$ To the best of our knowledge Kim and coworkers have reported the only Q7-fluorophore conjugate. ${ }^{28}$ Their Q7-Cy3 conjugate was used to detect vesicle fusion via fluorescence resonance energy transfer (FRET) between Cy3 and its FRET partner, Cy5, which was conjugated to a high affinity guest, adamantane ammonium. Their conjugate, however, was 
not studied in a living system. In parallel with these efforts, we describe here the synthesis of a conjugate between Q7 and tetramethylrhodamine (TMR) and the characterization of its optical, guest binding, and cellular uptake properties.

\section{RESULTS AND DISCUSSION}

Design and Synthesis. Our conjugate design took into account several considerations. A complementary pair of mono-functionalized Q7 and mono-functionalized TMR were chosen to ensure a single point of conjugation to Q7. A Hüisgen 1,3-dipolar cycloaddition ${ }^{29}$ was chosen to make use of a readily available monofunctional azidobutyl $\mathrm{Q}^{23}$ and to avoid amine-based coupling strategies (Q7 binds amines). A strained dibenzocyclooctyne (DBCO) linker was chosen to avoid Cu-based catalysts, ${ }^{30}$ which may also bind Q7. A dye with modest affinity for Q7 was chosen to limit the competition with target analytes.

Based on these considerations, the 6-isomer of the Q7-TMR conjugate (Q7R) was synthesized in two steps from monofunctional azidobutyl Q7 $\left(\mathrm{Q} 7-\mathrm{N}_{3}\right)^{23}$ (Figure 1) (see Supporting Information for experimental details). First, an equimolar quantity of 5/6-NHS-TMR was treated with the DBCO-amine crosslinker in Hünig's base to produce a mixture of 6-DBCO-rhodamine (6DR) and its constitutional isomer (5DR), which were obtained in 13\% yield each following HPLC purification. The identities of the 5DR and 6DR were confirmed by HPLC co-injection with authentic samples synthesized from the more expensive 5-NHS-TMR or 6-NHS-TMR isomers (Figure S2). Coupling of 6DR with Q7-N 3 was performed in 20\% aqueous DMSO to yield a 1:1 mixture of the 1,4- and 1,5-substituted triazole isomers of Q7R (Figures S7-S8) in 45\% recovery following HPLC purification. This mixture of triazole isomers of the 6-substituted Q7R conjugate was used for all subsequent studies. ${ }^{31}$

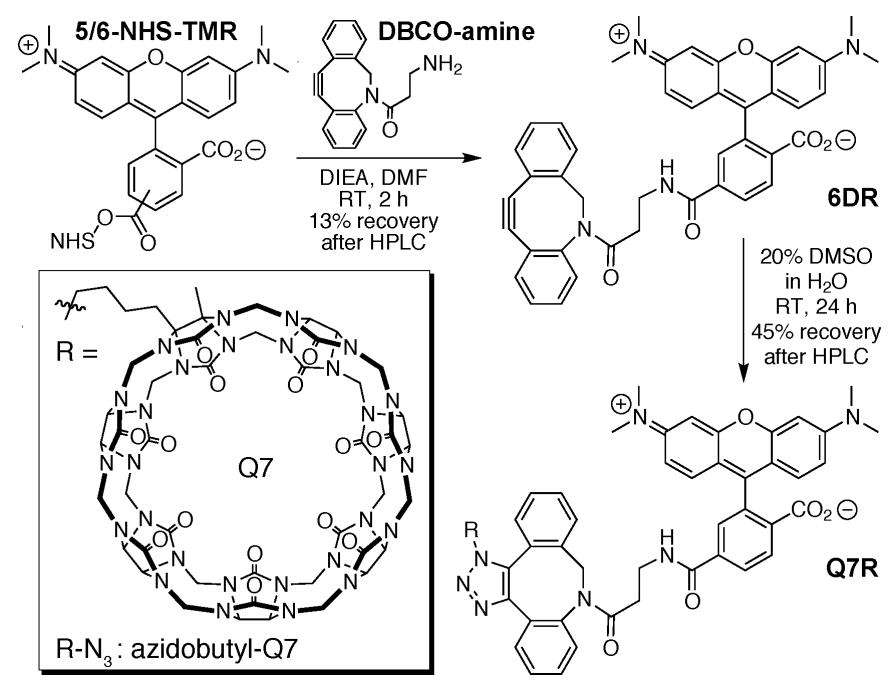

Figure 1. Synthesis of the cucurbit[7]uril-tetramethylrhodamine conjugate (Q7R). 
Solubility and Optical Properties. Among the cucurbit[n]uril homologues, Q7 displays the highest aqueous solubility $(20-30 \mathrm{mM}) .{ }^{10}$ Accordingly, it has been used as a solubilizer for hydrophobic pharmaceuticals, ${ }^{32}$ and it has been shown to enhance the solubility of free rhodamine. ${ }^{33}$ As expected, the solubility of Q7R in pure water at room temperature $(860 \pm 20 \mu \mathrm{M})$ was 45 -fold greater than that observed for TMR $(19 \pm 3 \mu \mathrm{M})$, demonstrating the solubilizing ability of the Q7 group. Despite the measured solubility of Q7R near $1 \mathrm{mM}$, we observe the slow formation of Q7R precipitate at high $\mu \mathrm{M}$ concentrations, thus necessitating the acquisition of NMR spectra at $150 \mu \mathrm{M}$. Even at this concentration, the spectrum of Q7R shows significant peak broadening, perhaps due to the formation of soluble aggregates. The addition of excess guest reduces peak broadening significantly (Figure S9).

As a baseline for subsequent binding studies, absorption and fluorescence spectra of Q7R were acquired in the absence of guest (Figures S10-S11). The visible absorbance spectrum of Q7R was nearly identical to that of tetramethylrhodamine ethyl ester (TMRE). A $3 \mathrm{~nm}$ blue-shift in the wavelength of maximum absorbance $\left(\lambda_{\max }\right)$ of Q7R relative to that of TMRE was observed. Similar peak shifts have been reported for xanthene dyes upon binding to Q7. ${ }^{1,32,34}$ The fluorescence intensity of Q7R, however, was significantly higher than that of TMRE, as would be expected for binding of the dye to Q7. ${ }^{1}$

Guest Binding. Guests 1-4 (Figure 2) were selected for binding studies because they bind to Q7 with $\mathrm{K}_{\mathrm{d}}$ values that span the useful $\mu \mathrm{M}$ to $\mathrm{nM}$ range. ${ }^{15,35,36}$ The addition of guest to Q7R resulted in no change in Q7R absorbance, but the fluorescence intensity of Q7R was quenched significantly in the presence of each guest (Figure 3a). Using fluorescence quenching as an observable for guest binding, we carried out equilibrium binding titrations for guests 1-4 in $10 \mathrm{mM}$ sodium phosphate buffered water, $\mathrm{pH}$ 7.0. In all cases, concentration-dependent quenching of Q7R fluorescence was observed (Figures $3 \mathrm{~b}$ and S17-S19). The data were fit to a simple 1:1 host $\bullet$ guest equilibrium binding model to determine $\mathrm{K}_{\mathrm{d}}$ values (Table 1). Complex stoichiometry was confirmed to be 1:1 (host:guest) by isothermal titration calorimetry and mass spectrometry experiments (Figures S12-S16). ${ }^{37}$

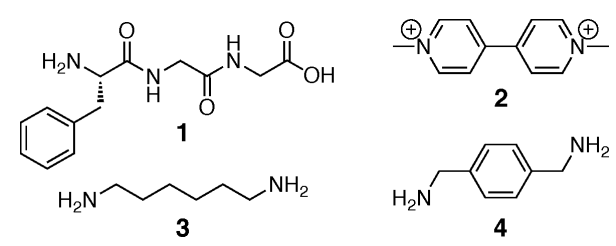

Figure 2. Formulas of the guests used in this study. 

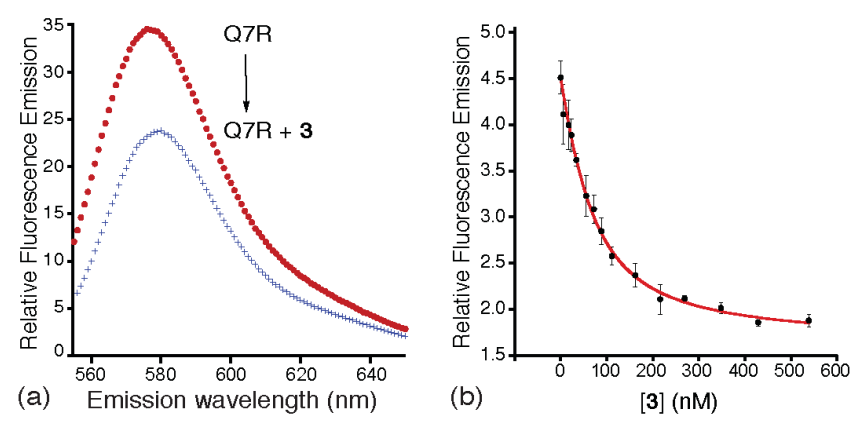

Figure 3. Representative fluorescence data for the binding of Q7R with guest 3 at $25{ }^{\circ} \mathrm{C}$ in $10 \mathrm{mM}$ sodium phosphate buffer, pH 7.0. (a) Fluorescence emission spectra of Q7R in the presence $(g r e y+$ ) and absence (red •) of 3. (b) Titration of Q7R $(50 \mathrm{nM})$ with 3, showing the fluorescence emission intensities at $577 \mathrm{~nm}\left(\lambda_{\mathrm{ex}}=550 \mathrm{~nm}\right)$ as a function of total guest concentration. Data points are average values from three experiments; error bars are standard deviations. The red line is the best fit to a binary equilibrium binding model.

Table 1. Binding constants of Q7R and Q7 with 1-4.

\begin{tabular}{lll}
\hline Guest & Q7R K $_{d}(\mathrm{M})^{\mathrm{a}}$ & $\mathrm{Q}^{\mathrm{Q}} \mathrm{K}_{\mathrm{d}}(\mathrm{M})^{\mathrm{b}}$ \\
\hline $\mathbf{1}$ & $3.6( \pm 1.0) \times 10^{-7}$ & $3.6( \pm 0.1) \times 10^{-7}$ \\
$\mathbf{2}$ & $1.6( \pm 1.8) \times 10^{-7}$ & $1.5( \pm 0.1) \times 10^{-7}$ \\
$\mathbf{3}$ & $4.6( \pm 1.8) \times 10^{-8}$ & $1.1( \pm 0.2) \times 10^{-8}$ \\
$\mathbf{4}$ & $6.5( \pm 2.7) \times 10^{-10}$ & $5.4( \pm 1.0) \times 10^{-10}$
\end{tabular}

${ }^{a}$ Fluorescence titrations were performed at $25^{\circ} \mathrm{C}$ in $10 \mathrm{mM}$ sodium phosphate, $\mathrm{pH} 7.0$, at $\lambda_{\mathrm{ex}}=$ $550 \mathrm{~nm}$ and $\lambda_{\mathrm{em}}=577 \mathrm{~nm}$ with Q7R concentrations fixed at 1000, 600, 50, and $10 \mathrm{nM}$ for titrations with $\mathbf{1}, \mathbf{2}, \mathbf{3}$, and $\mathbf{4}$, respectively. ${ }^{\mathrm{b}}$ Reported literature values for Q7. ${ }^{15,35,36}$

Initially, we were puzzled that guests 1-4 bind to Q7R and to free Q7 with essentially identical $K_{d}$ values. We were cognizant that prior work with Q7 and free TMR established a $\mathrm{K}_{d}$ value of $71 \mu \mathrm{M}$ for the Q7•TMR complex and an increase in the quantum yield of fluorescence upon complex formation. ${ }^{1,34}$ Given that our binding assays were conducted at concentrations well below the $\mathrm{K}_{\mathrm{d}}$ for intermolecular association, we surmised that the observed fluorescence quenching of Q7R upon guest binding was due to competitive displacement of the TMR group of Q7R from the cavity of Q7 within a self-complexed Q7R molecule. Theory tells us that the observed $\mathrm{K}_{\mathrm{d}}$ values for guest binding must be reduced by the intramolecular self-association constant, which again was puzzling until we realized that an intramolecular self-association constant near unity could explain the observed behavior. Accordingly, we hypothesize that Q7R exists in two nearly isoenergetic conformations that possess very different fluorescence 
properties; we do not believe the TMR group of Q7R is bound significantly within the Q7 cavity but may be loosely associated near the $\mathrm{C}=\mathrm{O}$ portals. Binding of a guest inside the $\mathrm{Q} 7$ cavity of $\mathrm{Q} 7 \mathrm{R}$ then favors the conformation with the lower fluorescence intensity. We believe this represents a new and potentially general strategy for the design of single-component host-indicator conjugates that respond sensitively to analytes without perturbing the innate molecular recognition properties of the host.

Imaging. In addition to sensing applications, covalent host-indicator conjugates should also be preferable to noncovalent host-indicator complexes for imaging applications. The covalent connection ensures that the indicator is attached to the host and that the host is being observed directly. Moreover, the lack of free dye reduces background signal. ${ }^{2}$ To assess compatibility with cell imaging applications, we treated HT22 neurons with Q7R and observed cellular uptake and punctate localization to the cytoplasm via live cell (Figure 4) and fixed cell (Figure S20a) confocal fluorescence microscopy. Live cell imaging with organelle markers (Figure S22) shows no pattern of localization to endoplasmic reticulum, golgi apparatus, lysosomes, or mitochondria. TMRE was not visibly cell permeable (Figure S20b) at the same concentration as Q7R $(91 \mathrm{nM})$, and therefore the Q7 group was required for uptake. Excess unlabeled Q7 did not inhibit the uptake of Q7R (Figure S21), and therefore receptor-mediated transport is likely not involved. Although not confirmed unambiguously, we believe that Q7R internalizes via endocytosis and localizes to endosomes. As might be expected from prior work on tolerance to Q7, ${ }^{38}$ the cells showed no perturbation in morphology or confluency from treatment with Q7R at concentrations up to $2.2 \mu \mathrm{M}$ over four days, and therefore Q7R did not appear to alter cell growth (Figures S23-S26).

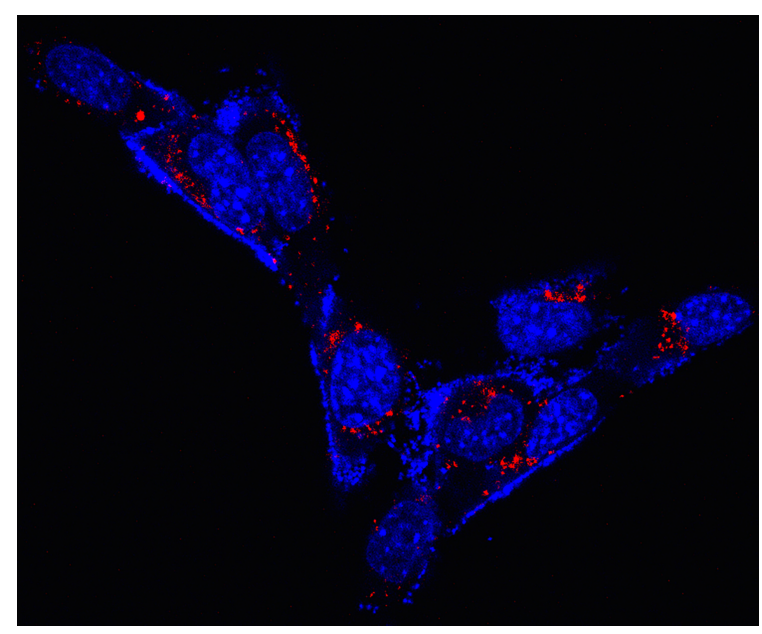

Figure 4. False color confocal fluorescence micrograph of live HT22 cells treated with $91 \mu \mathrm{M}$ Q7R (red) and Hoechst 33342 (blue). 


\section{CONCLUSIONS}

This paper describes the design, synthesis, and characterization of the guest binding and cell uptake of a single-component, direct optical sensor based on Q7. The Q7-rhodamine conjugate, Q7R, displays concentration-dependent fluorescence quenching upon binding to guests, and yet its binding affinities match those of unmodified Q7. The covalent attachment of host and indicator allowed us to measure $\mathrm{K}_{\mathrm{d}}$ values across three orders of magnitude. The ability to measure guest binding with unmitigated affinity allowed us to directly determine $\mathrm{K}_{\mathrm{d}}$ values at concentrations as low as $0.7 \mathrm{nM}$. These properties demonstrate the versatility of this compound for sensing applications and the use of Q7R as a Q7 surrogate for the direct measurement of guest binding over a wide range of concentrations. This work presages the use of Q7R for continuous sensing applications and as a single component reporter for supramolecular enzyme assays. $^{39}$

\section{ASSOCIATED CONTENT}

\section{Supporting Information}

The Supporting Information includes experimental details and optical, NMR, and mass spectra. It is available free of charge on the ACS Publications website.

\section{AUTHOR INFORMATION}

\section{Corresponding Author}

* Email: aurbach@trinity.edu

\section{Notes}

The authors declare no competing financial interest.

\section{ACKNOWLEDGMENT}

We gratefully acknowledge support from the Welch Foundation (W-0031 and W-1640) and the National Science Foundation (CHE-1309978 to A. R. U., CHE-1404911 to L. I., and DBI-1229702 to J. L. R.). O. A. A. was a Beckman Scholar. B. V. thanks the Department of Education for a GAANN fellowship (P200A120241) and the University of Maryland (Millard and Lee Alexander Fellowship) for support. 


\section{REFERENCES}

1. Dsouza, R. N.; Pischel, U.; Nau, W. M. Chem. Rev. 2011, 111, 7941.

2. You, L.; Zha, D.; Anslyn, E. V. Chem. Rev. 2015, 115, 7840-7892 .

3. Houk, K. N.; Leach, A. G.; Kim, S. P.; Zhang, X. Angew. Chem. Int. Ed. 2003, 42, 4872.

4. Ueno, A.; Kuwabara, T.; Nakamura, A.; Toda, F. Nature 1992, 356, 136.

5. Kim, J. S.; Quang, D. T. Chem. Rev. 2007, 107, 3780.

6. Ogoshi, T.; Harada, A. Sensors 2008, 8, 4961.

7. Azov, V. A.; Schlegel, A.; Diederich, F. Bull. Chem. Soc. Japan 2006, 79, 1926.

8. Ogoshi, T.; Yamagishi, T.-A. Eur. J. Org. Chem. 2013, 2961.

9. Kim, J.; Jung, I.-S.; Kim, S.-Y.; Lee, E. Kang; J.-K., Sakamoto; S., Yamaguchi, K.; Kim, K. J. Am. Chem. Soc. 2000, 122, 540.

10. Lee, J. W.; Samal, S.; Selvapalam, N.; Kim, H. J.; Kim, K. Acc. Chem. Res. 2003, 36, 621.

11. Lagona, L.; Mukhopadhyay, P.; Chakrabarti, S.; Isaacs, L. Angew. Chem. Int. Ed. 2005, 44, 4844.

12. Masson, E.; Ling, X.; Joseph, R.; Kyeremeh-Mensah, L.; Lu, X. RSC Adv. 2012, 2, 1213.

13. Barrow, S. J.; Kasera, S.; Rowland, M. J.; del Barrio, J.; Scherman, O. A. Chem. Rev. 2015, 115, 12320.

14. Biedermann, F.; Nau, W. M.; Schneider, H.-J. Angew. Chem. Int. Ed. 2014, 53, 11158.

15. Liu, S.; Ruspic, C.; Mukhopadhyay, P.; Chakrabarti, S.; Zavalij, P. Y.; Isaacs, L. J. Am. Chem. Soc. 2005, 127, 15959.

16. Rekharsky, M. V.; Mori, T.; Yang, C.; Ko, Y. H.; Selvapalam, N.; Kim, H., Sobransingh, D.; Kaifer, A. E.; Liu, S.; Isaacs, L.; Chen, W.; Moghaddam, S.; Gilson, M. K.; Kim, K.; Inoue, Y. Proc. Natl. Acad. Sci. USA 2007, 104, 20737.

17. Shetty, D.; Khedkar, J. K.; Park, K. M.; Kim, K. Chem. Soc. Rev. 2015, 44, 8747.

18. Cao, L.; Sekotur, M.; Zavalij, P. Y.; Mlinaric-Majerski, M.; Glaser, R.; Isaacs, L. Angew. Chem. Int. Ed. 2014, 53, 988.

19. Nau, W. M.; Scherman, O. A. Isr. J. Chem. 2011, 51, 492.

20. Walker, S.; Oun, R.; McInnes, F. J.; Wheate, N. J. Isr. J. Chem. 2011, 51, 616.

21. Assaf, K. I. \& Nau, W. M. Chem. Soc. Rev. 44, 394-418 (2014).

22. Kim, K.; Selvapalam, N.; Ko, Y. H.; Park, K. M.; Kim, D.; Kim, J. Chem. Soc. Rev. 2007, 36, 267.

23. Vinciguerra, B.; Cao, L.; Cannon, J. R.; Zavalij, P. Y.; Fenselau, C.; Isaacs, L. J. Am. Chem. Soc. 2012, 134, 13133. 
24. Ahn, Y.; Jang, Y.; Selvapalam, N.; Yun, G.; Kim, K. Angew. Chem. Int. Ed. 2013, 52, 3140.

25. Zhang, M.; Cao, L.; Isaacs, L. Chem. Commun. 2014, 50, 14756.

26. Ayhan, M. M.; Karoui, H.; Hardy, M.; Rockenbauer, A.; Charles, L.; Rosas, R.; Udachin, K.;

Tordo, P.; Bardelang, D.; Ouari, O. J. Am. Chem. Soc. 2015, 137, 10238.

27. Yu, Y.; Li, J.; Zhang, M.; Cao, L.; Isaacs, L. Chem. Commun. 2015, 51, 3762.

28. Gong, B.; Choi, B.-K.; Kim, J.-Y.; Shetty, D.; Ko, Y. H.; Selvapalam, N.; Lee, N. K.; Kim, K. J. Am. Chem. Soc. 2015, 137, 8908 .

29. Kolb, H. C.; Finn, M. G.; Sharpless, K. B. Angew. Chem. Int. Ed. 2001, 40, 2004.

30. Jewett, J. C.; Bertozzi, C. R. Chem. Soc. Rev. 2010, 39, 1272.

31. It is worth noting that the Q7-rhodamine conjugate obtained from the 5-NHS-TMR isomer does not experience a change in fluorescence upon treatment with guests. It may be that the TMR moiety does not adopt a conformation that responds to (e.g., competes with) guest binding, or it may be that upon guest binding, the TMR moiety does not undergo a change in its quantum yield of fluorescence.

32. Robinson, E. L.; Zavalij, P. Y.; Isaacs, L. Supramol. Chem. 2014, 27, 288.

33. Mohanty, J.; Nau, W. M. Angew. Chem. Int. Ed. 2005, 44, 3750.

34. Nau, W. M.; Mohanty, J. Int. J. Photoenergy 2005, 7, 133.

35. Vincil, G. A.; Urbach, A. R. Supramol. Chem. 2008, 20, 681.

36. Chinai, J. M.; Taylor, A. B.; Ryno, L. M.; Hargreaves, N. D.; Morris, C. A.; Hart, P. J.; Urbach, A. R. J. Am. Chem. Soc. 2011, 133, 8810.

37. For comparison, the ITC data for the complex of Q7R with compound 2 yielded a binding constant of $6.3( \pm 0.1) \times 10^{-7} \mathrm{M}$. This value is slightly weaker than that observed by fluorescence titration, which is due to some extent to the higher temperature in the ITC experiment.

38. Uzunova, V. D.; Cullinane, C.; Brix, K.; Nau, W. M.; Day, A. I. Org. Biomol. Chem. 2010, 8, 2037.

39. Dsouza, R. N.; Hennig, A.; Nau, W. M. Chem. Eur. J. 2012, 18, 1521.

\section{Table of Contents Graphic:}

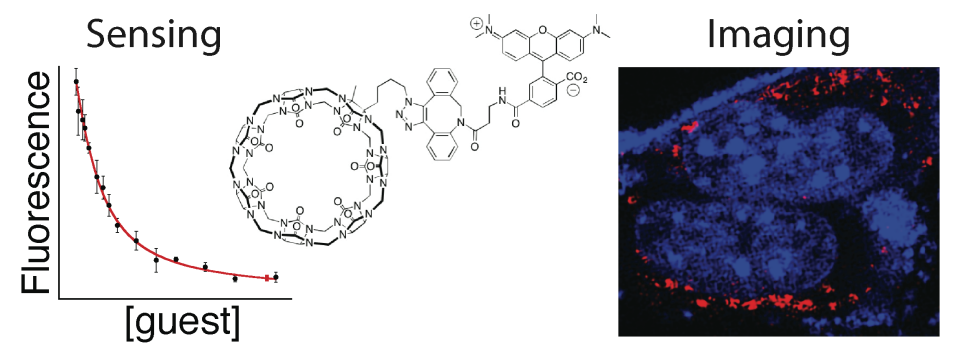

